\title{
EFEITOS DA FOTOBIOMODULAÇÃO NAS ALTERAÇÕES EXTRAPULMONARES OCASIONADAS PELO ENFISEMA EXPERIMENTAL EM Rattus norvegicus
}

\section{Khetyma Moreira Fonseca Leonardo de Melo Rodrigues}

Discentes da Faculdade de Ciências Médicas (FACIME), Teresina, PI, Brasil

\section{Danniel Cabral Leão Ferreira}

Universidade Federal do Piauí (UFPI), Teresina, PI, Brasil.

E-mail: ketyma_mf@hotmail.com

Márcia Milena Oliveira Vilaça

Associação de Ensino Superior do Piauí (AESPI).

\section{Suzana Maria Pereira Galvão}

Docente na Universidade Estadual do Piauí (UESPI), Teresina, PI, Brasil.

Wellington dos Santos Alves

Docente na Universidade Estadual do Piauí (UESPI) e na Faculdade Santo Agostinho, Teresina, PI, Brasil. .
RESUMO: O enfisema pulmonar é um processo inflamatório pulmonar e tem repercussões extrapulmonares. Os objetivos desta pesquisa são investigar os efeitos da fotobiomodulação nas alterações extrapulmonares. Foram selecionados 24 ratos, divididos em três grupos de 8 animais: grupo controle (GC: recebeu apenas solução salina); grupo DPOC (GD: instilação de papaína $2 \mathrm{mg} / \mathrm{kg}$ ); grupo DPOC + laser (GDL: instilação de papaína + 15 dias de laserterapia). Para análise dos dados foi coletado e observado: $\mathrm{CK}_{\mathrm{MB}}$, $\mathrm{LBA}$, Histologia (análise morfométrica) e espessura das câmaras cardíacas (análise morfométrica), respectivamente. Houve diminuição no número de células inflamatórias no LBA do GDL $(p<0,05)$ quando comparado com GC e GD; aumento dos níveis de $\mathrm{CK}_{\mathrm{MB}}$ no grupo GD $(p<0,05)$; e diminuição significativa no GDL $(p=0,0009)$ quando comparado com o GD, hipertrofia ventricular esquerda $(p<0,05)$ no grupo GD e GDL quando comparado com controle. A fotobiomodulação diminui a liberação de células inflamatórias responsáveis pela liberação do $\mathrm{CK}_{\mathrm{MB}}$ na corrente sanguínea, diminuindo assim os níveis de $\mathrm{CK}_{\mathrm{MB}}$ e reduzindo os riscos de infarto do miocárdio.

PALAVRAS-CHAVE: Enfisema; Fisiopatologia; Modelos Animais; Papaína; Terapia a Laser de Baixa Intensidade.

\section{EFFECT OF PHOTOBIOMODULATION IN EXTRAPULMONARY CHANGES CAUSED BY EXPERIMENTAL EMPHYSEMA IN Rattus norvegicus}

ABSTRACT: Lung emphysema is a pulmonary inflammatory process, with extrapulmonary repercussions. Twenty-four rats were selected and divided into three groups with eight animals each: control (GC: received only saline solution); group DPOC (GD: with instillation of papaya proteinase $2 \mathrm{mg} / \mathrm{kg}$ ); group DPOC + laser (GDL: with instillation of papaya proteinase +15 days of laser therapy). Data analysis comprised: $\mathrm{CK}_{\mathrm{MB}}$, LBA, Histology (morphometric analysis) and thickness of the cardiac chambers (morphometric analysis), respectively. The number of inflammatory cells in LBA of GDL decreased $(p<0.05)$ when compared with GC and GD; there was an increase in $\mathrm{CK}_{\mathrm{MB}}$ levels in group GD $(p<0.05)$; significant decrease in GDL $(p=0.0009)$ when compared to GD, left ventricle hypertrophy $(p<0.05)$ in group GD and GDL when compared to control. Photobiomodulation decreased the release of inflammatory cells by the release of $\mathrm{CK}_{\mathrm{MB}}$ in the blood and decreased $\mathrm{CK}_{\mathrm{MB}}$ levels and reduced myocardial infarction risks.

KEY WORDS: Emphysema; Physiopathology; Animal Models; Papaya Proteinase; Low Intensity Laser Therapy. 


\section{INTRODUÇÃO}

A doença pulmonar obstrutiva crônica (DPOC) é uma enfermidade respiratória cujo principal fenótipo é o enfisema pulmonar (ISHI et al., 2011). O enfisema pulmonar é uma doença que desencadeia a obstrução do fluxo aéreo progressivamente e está associada a uma resposta inflamatória anormal dos pulmões decorrente da inalação de partículas ou gases tóxicos e tem consequências sistêmicas significativas (GOLD, 2011). A DPOC é um problema de saúde de grande magnitude não apenas pela sua alta prevalência, mas devido às comorbidades associadas a este quadro elevando as taxas de mortalidade (CEZARE et al., 2010).

No Brasil vem ocorrendo paulatinamente um aumento do número de óbitos por DPOC nos últimos 20 anos, pois é considerado um dos países da América Latina que mais desembolsam verbas anualmente para a assistência do paciente com DPOC, sendo superado apenas pela Argentina, Equador e Venezuela. Esta doença apresenta elevado custo socioeconômico, uma vez que os DPOCíticos são lentamente afastados do seu cotidiano por decorrência das disfunções musculoesqueléticas que diminuem a sua capacidade respiratória. Além de acometer os pulmões, a DPOC tem manifestações sistêmicas a qual repercutem de maneira importante sobre a qualidade de vida e a sobrevida dos pacientes (BARNES; CELLI, 2009).

Há estudos que comprovem a repercussão além do sistema pulmonar no paciente enfisematoso; dentre essas alterações pode-se citar doença da artéria coronariana ou insuficiência cardíaca congestiva e a cor pulmonale, insuficiência cardíaca, arritmias cardíacas e disfunção ventricular direita que podem ser comorbidades associadas a esta doença (de DIEZ et al., 2010).

O enfisema pulmonar gera extrapulmonarmente quadros de hipoxemia e, até mesmo, anóxia levando a alterações hidroeletrolíticas e enzimáticas, decorrentes da liberação do conteúdo de células danificadas dentro da circulação sistêmica (RUFINO; DA COSTA, 2013). As prováveis células e enzimas liberadas são os neutrófilos, macrófagos, creatina fosfoquinase (CPK), proteína C reativa (PCR), estas possuem importância clínica e experimental, visto que a sua análise pode ser um parâmetro para se estimar a viabilidade muscular, neste caso, do músculo cardíaco (GARCIA et al., 2015).

No estudo realizado por Seimetz et al. (2011) foi denotado que, além do comprometimento pulmonar irreversível desencadeado pelo enfisema experimental, havia disfunção pulmonar, remodelamento vascular e hipertensão pulmonar. Outro estudo que descreve alterações extrapulmonares também é o de Icochea; Cooper; Kuhn (2010), que retratam a cor pulmonale, a qual modificou a morfometria das câmaras cardíacas e a sua relação entre a hipoxemia desencadeada pelo enfisema experimental em camundongos. Vários modelos de enfisema pulmonar têm sido desenvolvidos como ferramenta de investigação para os fatores bioquímicos e fisiopatológicos responsáveis pela evolução da doença (MONTEIRO et al., 2004).

A terapia de primeira escolha para o enfisema é à base de corticosteroides, oxigenoterapia, dentre outras estratégias, visando a qualidade de vida do paciente (PRICE et al., 2013). Entretanto, existe outra estratégia bastante promissora que ainda está sob evidenciação científica que é a fotobiomodulação por meio da laserterapia de baixa potência, que tem efeitos positivos quanto à modulação do processo inflamatório (RIBEIRO et al., 2016).

A fotobiomodulação por meio da laserterapia propiciaváriosbenefícios, dentreelesaaçãoantiedematosa, anti-inflamatória e cicatricial, pois ocasiona aumento da pressão hidrostática no capilar pulmonar e eliminação de catabólitos, aumenta a microcirculação local e aumenta a celularidade dos tecidos na região irradiada (OLIVEIRA JÚNIOR et al., 2010).

Segundo Karu (1999), a laserterapia tem efeito anti-inflamatório, pois atua inibindo a ação do Fator de Necrose Tumoral (TNF- $\alpha$ ) e Interleucina (IL- $\beta$ ), o que interrompe a quimiotaxia celular para o pulmão, no enfisema experimental, principalmente na fase inicial do processo inflamatório diminuindo, assim, os números de neutrófilos, macrófagos e linfócitos.

Com base na literatura pesquisada percebeuse que não há relatos de estudos que correlacionem as alterações extrapulmonares decorrentes do enfisema experimental com os efeitos da fotobiomodulação por meio do laser de baixa potência, pois a fotobiomodulação 
nas alterações extrapulmonares poderá ser benéfica, já que tem efeito inibidor da cascata inflamatória, como desencadeada pela cor pulmonale, por exemplo, onde há aumento de TNF- $\alpha$ na circulação (LIAO et al., 2012).

Diante disso, faz-se necessário conduzir estudos sobre os possíveis benefícios da fotobiomodulação nas alterações morfométricas, enzimáticas e inflamatórias decorrentes do enfisema experimental nas alterações extrapulmonares, neste caso, no sistema cardíaco.

\section{MATERIAL E MÉTODO}

\subsection{METODOLOGIA COM ANIMAIS}

Foram utilizados ratos (Rattus norvegicus, albinus, Wistar) com três meses de vida e peso médio de $300 \mathrm{~g} \pm 20 \mathrm{~g}$, provenientes do Biotério do Centro de Ciências da Saúde da Universidade Estadual do Piauí (CCS-UESPI). Os animais foram mantidos sob condições livres de organismos patogênicos com acesso livre a ração e água "ad libitum" e ciclo claro/escuro de 12 horas e temperatura de $23^{\circ} \mathrm{C} \pm 1{ }^{\circ} \mathrm{C}$. O experimento com animais foi realizado após aprovação pelo Comitê de Ética em Pesquisa Animal da Universidade Estadual do Piauí (CEUA UESPI $n^{0}$ 05733/15) e foi desenvolvido em conformidade com as diretrizes do Conselho Nacional de Controle de Experimentação Animal (CONCEA). Para anestesia foram administradas doses contendo ketamina $\left(\right.$ Dopalen $\left.^{\circledR}\right)\left(100 \mathrm{mg} / \mathrm{kg}\right.$, ip) e xilazina (Anasedan $\left.{ }^{\circledR}\right)(20$ $\mathrm{mg} / \mathrm{kg}$, ip). Para a eutanásia foi administrada superdose intraperitonealmente de $100 \mathrm{mg} / \mathrm{kg}$ de Tiopental ${ }^{\circledR}$.

\subsection{GRUPOS EXPERIMENTAIS}

Foram utilizados 24 ratos Wistar, divididos em 3 grupos com 08 animais cada: grupo controle (GC: que recebeu solução salina); grupo DPOC (GD: dose única de papaína $0,2 \mathrm{mg} / \mathrm{kg}$ ); e grupo DPOC + Laser (GDL: papaína $0,2 \mathrm{mg} / \mathrm{kg}+$ laser $)$.

\subsection{INDUÇÃO DO ENFISEMA}

A indução do enfisema pulmonar por papaína foi realizada pela via orotraqueal por meio de instilação de uma solução $(0,5 \mathrm{mg} / \mathrm{ml})$ dissolvida em solução fisiológica a $0,9 \%$, correspondendo a $0,2 \mathrm{mg} / \mathrm{kg}$ por animal (com base no protocolo de Fusco et al., 2002, modificado), com auxílio de sonda de aspiração $\mathrm{n}^{0} 4$ modificada e acoplada à bomba de infusão por volume Lifemed $^{\circledR}$ com taxa de infusão de $0,1 \mathrm{ml} / \mathrm{min}$.

\subsection{PROTOCOLO DE FOTOBIOMODULAÇÃO}

Após a indução do processo inflamatório os grupos GD e GDL ficaram sob observação durante 15 dias (sem receber nenhum tratamento) e o grupo GDL, a partir do $16^{\circ}$ dia após a aplicação da papaína, foi tratado com laser de baixa intensidade, comprimento de onda de $660 \mathrm{~nm}\left(\right.$ MMOpticos $\left.^{\circledR}\right)$ com potência de $10 \mathrm{~W}$, na dose de $12,5 \mathrm{~J} / \mathrm{cm}^{2}$ por 20 segundos/ponto, percutaneamente em 3 pontos: hemitórax direito, hemitórax esquerdo e na carina, durante 15 dias.

\subsection{DOSAGEM DE PROTEÍNA}

Os animais foram anestesiados para coletar uma amostra de sangue no tubo contendo gel anticoagulante diretamente da aorta abdominal com auxílio de seringa heparinizada onde se retirou no total $1 \mathrm{ml}$ de sangue que foi centrifugado para coleta do soro; após isto, foi realizada a análise enzimática da Creatina Fosfoquinase $\left(\mathrm{CK}_{\mathrm{MB}}\right)$ de acordo com a absorbância no espectrofotômetro ci8200 ${ }^{\circledR}$ que identificava concentrações mínimas de $25 \mu \mathrm{L}$ da enzima no soro.

\subsection{ANÁLISE DO LAVADO BRONCOALVEOLAR}

Após a coleta de sangue foi realizada uma incisão longitudinal na região cérvico-ventral com acesso à traqueia que foi exposta e canulada a $2 \mathrm{~cm}$ acima da carina com um escalpe que continha 2 vias: uma para injeção de $20 \mathrm{ml}$ de solução tampão (Fosfato de Sódio) nos pulmões e a outra para coleta do Lavado Brônquio Alveolar (LBA), contendo o tampão. Desta forma, após a injeção de PBS foram realizadas massagens de forma circular nos hemitórax direito e esquerdo para lavar o espaço bronco alveolar e, após isto, foi coletado o LBA. O LBA obtido foi para centrífuga (Baby Fanem ${ }^{\circledR}$ ) durante 10 minutos 
com $1500 \mathrm{rpm}$. O sobrenadante foi desprezado e o botão celular ressuspenso em $1 \mathrm{ml}$ de PBS, posteriormente foi colocada uma gota na lâmina de microscópio e colocada para secar na câmara escura; após isto, foi corada com kit de panotipo rápido, de acordo com as instruções do fabricante, para contagem diferencial de células inflamatórias com auxílio do contador de células $\left(\right.$ Digitamer $\left.^{\circledR}\right)$.

\subsection{ANÁLISE HISTOLÓGICA E MORFOMÉTRICA DO PULMÃO E CORAÇÃO}

Para a retirada do pulmão foi realizada uma perfusão cardíaca para a extração do sangue pulmonar. Após a eutanásia, os animais foram novamente acoplados à cânula. Através dessa, foi introduzido formaldeído a $10 \%$ tamponado. Os pulmões foram conservados por um período de 24 horas e, então, transferidos para recipientes contendo álcool etílico a $70 \%$, onde permaneceram por cerca de 72 horas. A preparação das lâminas foi de acordo com o protocolo de Silva (2003).

A quantificação do enfisema pulmonar foi verificada pela presença de destruição alveolar determinada medindo o diâmetro alveolar médio em micrômetros $(\mathrm{Lm})$. Esta técnica consiste em determinar o número de vezes que as estruturas do parênquima para a troca gasosa intercepta um conjunto de linhas coerentes. Assim, no caso de enfisema, o número de intercepções das estruturas alveolares com um sistema de linhas será menor, indicando destruição alveolar. O Lm - diâmetro médio alveolar é obtido pela equação:

$$
\mathrm{Lm}=\mathrm{Ltot} / \mathrm{Li}
$$

Onde:

Ltot é o comprimento total de linhas no campo microscópico;

e Li é o número de intercepções de estruturas alveolares com as linhas do retículo.

Com o objetivo de realizar a morfometria, cinco campos microscópicos, com aumento de 10x10 vezes, escolhidos aleatoriamente, sem sobreposição, foram fotografados e analisados usando uma retícula 100 pontos e 50 linhas sobrepostas à imagem do parênquima pulmonar.

Após a ressecção em bloco dos pulmões e corações, esses foram imersos no formol a $10 \%$ por 48 horas. Após a fixação dos mesmos, foi realizada a análise morfométrica das câmaras cardíacas por meio de fotografias dos ventrículos, através de uma secção transversal dos ventrículos no ponto médio na distância entre o ápice e o sulco coronário do coração, onde por meio do software Imagem Pro Plus ${ }^{\circledR}$ foi analisada espessura da parede do VD (aferido no ponto médio da parede livre), espessura da parede do VE (no ponto médio entre os músculos papilares craniais e caudais).

\subsection{ANÁLISE ESTATÍSTICA}

Os resultados obtidos foram submetidos à análise de variância (ANOVA) e, em seguida, ao teste Newman-Keuls. As análises estatísticas foram conduzidas utilizando o GraphPad Prism 5.0. Os resultados foram expressos como a média \pm erro-padrão da média (EPM). Valores de $p \leq 0,05$ foram considerados significativos.

\section{RESULTADOS}

Nos animais do grupo em que houve instilação de solução contendo papaína observou-se característica morfológica semelhante ao enfisema pan-acinar, ou seja, comprometimento dos ácinos com alargamento difuso compreendendo desde a região hilar até a periferia dos pulmões. Onde houve destruição dos septos com alargamento dos espaços alveolares, como visto na Figura 1B quando comparada com a Figura 1A. 


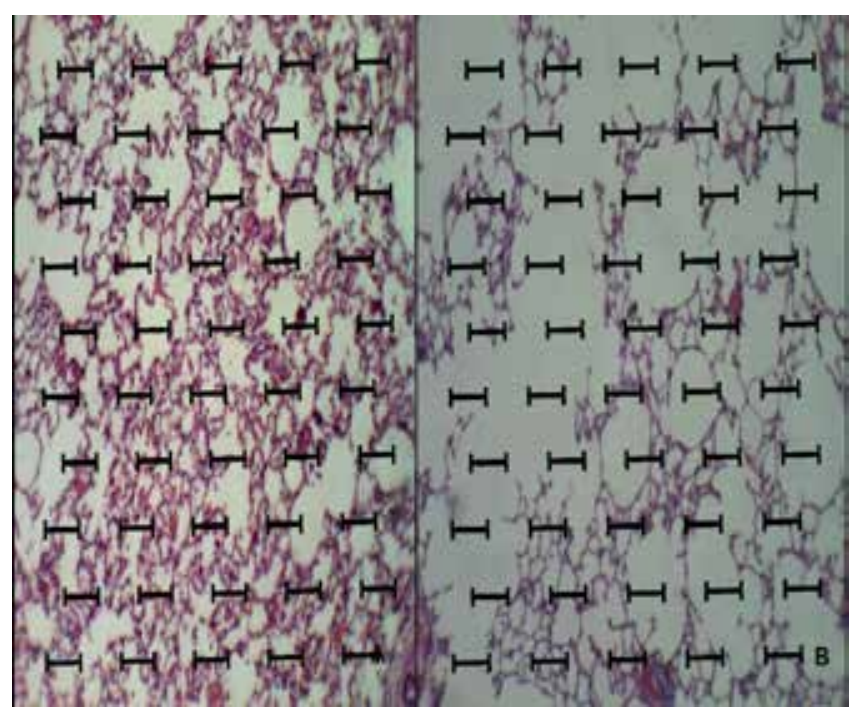

Figura 1. Fotomicrografias do parênquima pulmonar de ratos com retículo submetidos à instilação de solução fisiológica (A) e papaína (B). Coloração HE. Aumento de 100x

$\mathrm{O}$ aumento do diâmetro alveolar médio $\mathrm{e}$ consequente diminuição da área de superfície alveolar foi constatado nos dois pulmões dos animais pertencentes aos grupos que receberam doses de papaína $(2 \mathrm{mg} / \mathrm{kg})$ (Figura 2), demostrando a efetividade do protocolo de lesão.

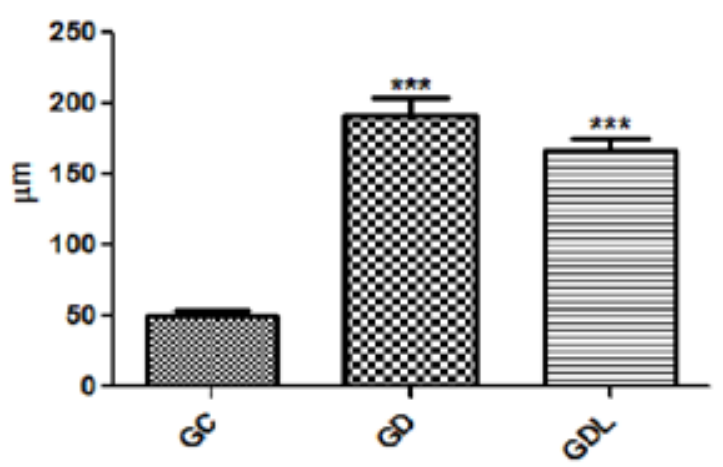

Figura 2. Diâmetro alveolar médio (em $\mu \mathrm{m}$ ), nos grupos GD, GL. Foi realizado teste Newman-Keuls usando o software GraphPad Prism, versão 5.01. *** $p \leq 0,05$ em relação ao GC.

Os grupos que receberam papaína GD e GL apresentaram um diâmetro alveolar maior que o grupo controle $(p \leq 0,05)$, sendo que o diâmetro alveolar médio do grupo controle, lesão e tratado, oscilaram entre 67 $\mu \mathrm{m}$ a $108 \mu \mathrm{m}, 125 \mu \mathrm{m}$ a $312 \mu \mathrm{m}$ e $138 \mu \mathrm{m}$ a $150 \mu \mathrm{m}$, respectivamente; quando se comparou o grupo tratado e lesão não se verificou diferença significativa quanto ao diâmetro alveolar $(p=0,5706)$, indicando que houve um estadiamento da lesão.

$\mathrm{Na}$ lesão por papaína, enzima proteolítica análoga à elastase, gera a destruição da histoarquitetura pulmonar, liberando células inflamatórias e mediadores relacionados ao quadro de enfisema (CAMPOS, 2015). A inflamação é mediada pelos mediadores químicos que promovem uma cadeia inflamatória com liberação de células inflamatórias como macrófagos, neutrófilos e eosinófilos (TARANTINO, 2008). Isto pode ser visto na Tabela 1.

Tabela 1. Contagem de células diferenciais no Lavado Bronco Alveolar

\begin{tabular}{llccc}
\hline Grupos & Células & Total & Média & $\begin{array}{c}\text { Desvio } \\
\text { Padrão }\end{array}$ \\
\hline \multirow{2}{*}{ GC } & Macrófagos & 610 & 61 & 17,5 \\
& Neutrófilos & 135 & 13,5 & 4,10 \\
& Eosinófilos & 43 & 4,3 & 2,83 \\
\hline \multirow{2}{*}{ GD } & Macrófagos & 876 & 87,6 & 4,07 \\
& Neutrófilos & 422 & 42,2 & 7,61 \\
& Eosinófilos & 53 & 5,3 & 4,26 \\
\hline \multirow{2}{*}{ GDL } & Macrófagos & 523 & 52,3 & 11,09 \\
& Neutrófilos & 88 & 8,8 & 2,18 \\
& Eosinófilos & 13 & 1,3 & 1,26 \\
\hline
\end{tabular}

Legenda: GC (Grupo controle); GD (Grupo DPOC); GDL (Grupo DPOC + Laser).

Fonte: Laboratório de Fisiologia Experimental da Universidade Estadual do Piauí (UESPI), Teresina, novembro de 2015.

No LBA pode-se notar aumento de células inflamatórias (macrófagos, neutrófilos e eosinófilos) quando comparado com grupo controle $(p \leq 0,05)$. Notase também em adição que houve diminuição significativa de células inflamatórias quando se compara os grupos GD e GDL $(p \leq 0,05)$ com o controle (Tabela 1). Quanto aos ventrículos direito a espessura foi similar entre os grupos GD, GDL e controle, com variações entre 1,09 a 1,59 $\mathrm{mm}, 1,01$ a $1,56 \mathrm{~mm}$ e 1,13 a 2,02 $\mathrm{mm}$ respectivamente, quando comparado com grupo controle não havendo resultado significativo $(\phi=0,9669)$, não havendo, portanto, indícios evidentes de cor pulmonale (Figura $3 \mathrm{~A})$. 

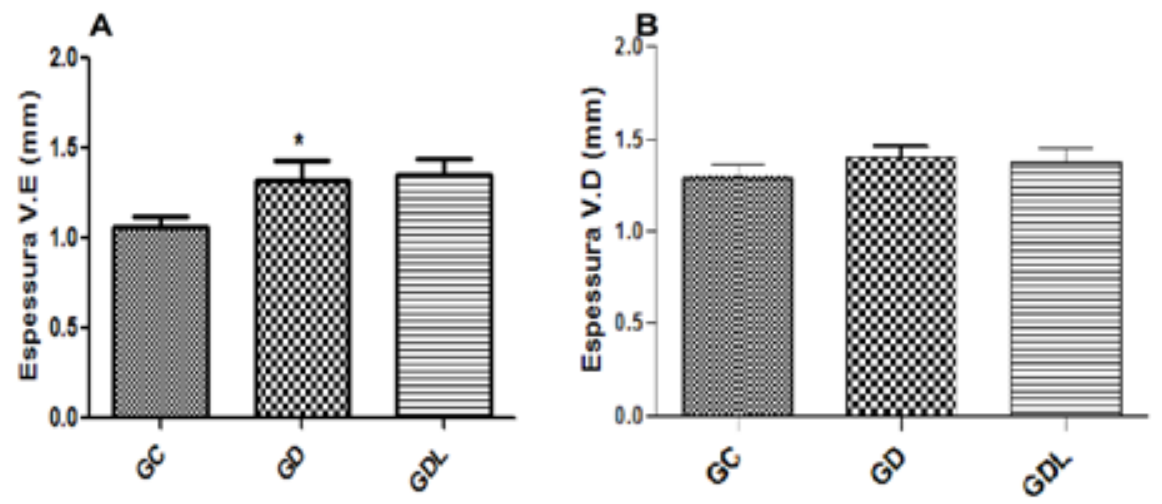

Figura 3. (A) Espessura do Ventrículo direito nos grupos GD e GD. (B) Espessura do Ventrículo esquerdo nos grupos GD e GDL. Foi realizado teste Newman-Keuls usando o software GraphPad Prism,versão 5.01. * $p \leq$ 0,05 confrontando com o GC.

A mensuração da espessura do ventrículo esquerdo mostrou aumento nos grupos de animais submetidos à instilação orotraqueal de papaína quando comparada com o grupo controle $(p=0,3055)$. Entretanto, quando se compara os grupos GD e GDL não houve resultado significado $(p=0,6711)$, denotando talvez a estabilização da espessura cardíaca (Figura 3B).

A Creatina Fosfoquinase é uma enzima encontrada em concentrações elevadas no tecido muscular cardíaco e esquelético e também em outros tecidos, sendo que Creatina Fosfoquinase $\left(\mathrm{CPK}_{\mathrm{MB}}\right)$ é específica do epitélio cardíaco sendo usado como marcador de lesão cardíaca quando está elevada. No grupo GD pode-se observar um aumento dos níveis de $\mathrm{CPK}_{\mathrm{MB}}$ estatisticamente significativo $(p<0,05)$ quando comparado com o controle. Entretanto, no grupo GDL houve uma diminuição significativa de $\mathrm{CPK}_{\mathrm{MB}}$ quando comparado com GD $(p<0,009)$ (Figura 4).

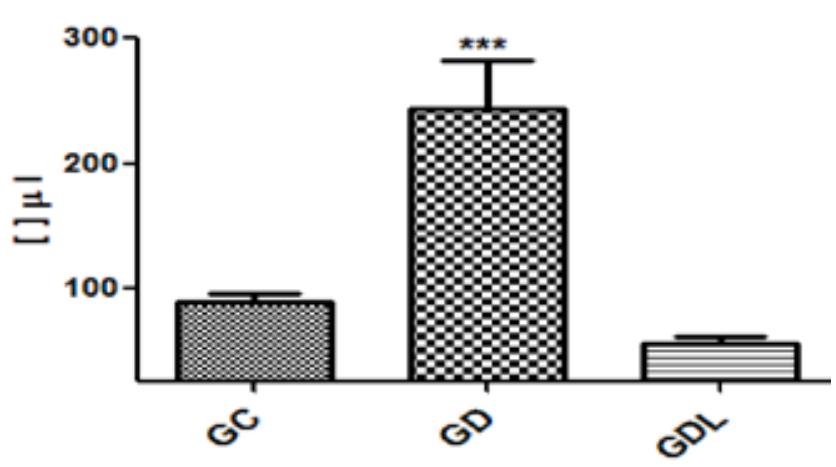

Figura 4. Concentração de Creatina Fosfoquinase $\left(\mathrm{CPK}_{\mathrm{mb}}\right)(\mathrm{em} \mu \mathrm{l})$ nos grupos (GC), (GD) e (GDL). Foi realizado teste Newman-Keuls usando o software GraphPad Prism, versão 5.01. ***p $\leq 0,05$.

\section{DISCUSSÃO}

Nos últimos 40 anos a pesquisa sobre 0 mecanismo de lesão parenquimatosa pulmonar in vivo por meio da administração de enzimas proteolíticas como a papaína, entre outras substâncias, causando enfisema, por exemplo, tem sido uma das áreas mais intensas de pesquisa sobre doenças pulmonares, visando novas formas de tratamento (OLIVEIRA JÚNIOR et al., 2010).

A papaína é reconhecida por apresentar uma potente atividade elastolítica, pois ela desencadeia alterações morfofuncionais características do enfisema. $\mathrm{Na}$ avaliação histopatológica por meio do cálculo do diâmetro alveolar nos pulmões dos animais submetidos ao protocolo de lesão com papaína, foi possível demonstrar que há uma destruição alveolar difusa, caracterizando um enfisema do tipo pan-acinar (Figura 1), onde o tipo panacinar é o mais prevalente em quase $95 \%$ dos casos de enfisema em humanos (MONTEIRO et al., 2004; FUSCO et al., 2002).

A utilização do cálculo do diâmetro alveolar empregado neste estudo é um método rotineiro utilizado para mensurar o enfisema pulmonar. Sabe-se que em ratos sem comprometimento pulmonar, o diâmetro alveolar médio é ao redor de $70 \mu \mathrm{m}$ (SULKOWSKA et al., 1995), sendo que no presente estudo, os grupos GD e GDL apresentaram diâmetro alveolar médio entre 125 $\mu \mathrm{m}$ a $150 \mu \mathrm{m}$, com valores estatisticamente significativos $(p \leq 0,05)$ comparando com o grupo controle. Tais dados denotam que nos pulmões houve ruptura significativa dos septos interalveolares com aumento importante dos 
espaços alveolares característicos do enfisema pan-acinar, visto também no estudo de Kuhl (2013).

Segundo Kozma (2012) as manifestações fisiopatológicas características do enfisema são resultado de um processo de inflamação crônica e estresse oxidativo persistente orquestrado primariamente pelos macrófagos (células-chave da imunidade nata).

Valença e Porto (2008) consideram também os macrófagos alveolares como representantes da primeira linha de defesa pulmonar e são responsáveis principalmente pela depuração pulmonar, pois, são capazes de captar, transportar e eliminar partículas por fagocitose. Estes macrófagos são ativados por fatores exógenos com a papaína, por exemplo, promovem a secreção de elastase, além de mediadores inflamatórios como TNF- $\alpha$, dentre outros mediadores sistêmicos que promovem a quimiotaxia e ativação de neutrófilos, amplificando o processo inflamatório como visto no Lavado Bronco alveolar (LBA), isto pode ser observado dos grupos GD e GDL quando comparados com o grupo controle $(p \leq 0,05)$ (Tabela 1).

Segundo descrito por Tarantino (2008), as células inflamatórias, principalmente os neutrófilos, são as células mais encontradas no LBA de pacientes com DPOC, além de ser considerada por alguns pesquisadores a principal célula envolvida na lesão elastolítica, assim como visto neste estudo quando comparado com os grupos GC, GD do estudo $(p \leq 0,05)$, conforme visto na Tabela 1 . Em adição, pode-se verificar também que no grupo GDL houve uma diminuição no número de neutrófilos no LBA $(p=0,0026)$ quando comparado com o grupo GD e GC.

Dentre as células inflamatórias encontradas observa-se a presença dos eosinófilos no LBA do enfisema. Alguns estudos, dentre eles o de Tarantino (2008), denotam que o aumento do número de eosinófilos nas vias aéreas de alguns pacientes com DPOC é decorrente do quadro de asma instalado concomitantemente. Entretanto, esta hipótese acima não foi observada, pois não houve alteração do número de eosinófilos no GD em comparação ao grupo controle.

Dentre as estratégias terapêuticas há uma que está em comprovação e se mostra bastante promissora, que é a laserterapia de baixa potência em virtude de seus efeitos anti-inflamatórios (OLIVEIRA JÚNIOR et al.,
2010). A laserterapia é uma estimulação tecidual usando a luz denominada de fotobioestimulação.

De acordo com Karu (1999), a bioestimulação através da fotobiomodulação em certas organelas ou membranas acelera a síntese de compostos comandados pelo DNA como os anti-inflamatórios que inibem a ação das IL-1 $\beta$ e TNF- $\alpha$, promovendo efeitos sistêmicos. Dentre os efeitos sistêmicos observados no estudo foi a diminuição de células inflamatórias encontradas no LBA, no grupo GDL quando comparado com os grupos GD e GC $(p \leq 0,05)$ (Tabela 1). Estes dados corroboram com os achados de Oliveira Junior et al. (2010).

Dentre as complicações descritas nos estudos com pacientes portadores de DPOC podemos citar a cor pulmonale, caracterizado pela dilatação e hipertrofia do ventrículo direito com potencial insuficiência secundária a hipertensão primaria, sendo que vários fatores contribuem para desencadear a cor pulmonale, dentre eles redução do leito vascular pulmonar, hipóxia tecidual e aumento da pressão arterial pulmonar (ICOCHEA; COOPER; KUHN, 2010).

Ainda que a cor pulmonale seja diagnosticada em pacientes com DPOC, não encontramos em nossos animais sinais de sua presença; este insucesso pode ser encontrado também no estudo de Monteiro (2004). Talvez seja consequência do protocolo de indução de lesão pulmonar por nós usado, apresentando grau de destruição alveolar insuficiente (Figura 3A). Além do mais, a cor pulmonale pode se desenvolver mais tardiamente, necessitando de um tempo maior de observação.

No presente estudo verificou-se a hipertrofia do ventrículo esquerdo (Figura 3B) quando comparamos os grupos GD e GDL com controle, tendo um resultado significativo $(p \leq 0,05)$. Este resultado pode ser observado por outros estudos que elucidam a presença da hipertrofia ventricular esquerda em pacientes com DPOC decorrente do aumento da pressão arterial sistêmica (SMITH et al., 2013). Outros pesquisadores, como Castardeli et al. (2005), observaram no modelo de lesão pulmonar que, por meio da inalação passiva de fumaça de cigarro por pelo menos dois meses, é induzida a remodelação cardíaca, caracterizada por alterações morfológicas e funcionais como a hipertrofia do ventrículo esquerdo também. 
Monteiro (2004) levantou uma hipótese para explicar tal achado: o mesmo baseia-se no princípio de que a lesão pulmonar ocasionada pela instilação traqueal de papaína pode induzir hipertensão arterial sistêmica. De acordo com os achados de Hansson et al. (2011), a HAS modifica a função endotelial, diminuindo a vasodilatação do endotélio e aumentando a interação de placas coronarianas e monócitos com as células endoteliais podendo evoluir com quadro de infarto agudo do miocárdio.

Segundo a Organização Mundial de Saúde (OMS), que cataloga como infarto agudo do miocárdio o indivíduo que apresenta pelo menos dois dos seguintes critérios: histórico de desconforto torácico tipo isquêmico, alterações em eletrocardiogramas seriados, compatíveis com a suspeita diagnóstica e, por último, o aumento e queda dos marcadores séricos cardíacos (RIBEIRO et al., 2012). Dentre os marcadores séricos podemos destacar a liberação de mediadores inflamatórios e da Creatina fosfoquinase específica da fibra cardíaca $\left(\mathrm{CK}_{\mathrm{MB}}\right)$, decorrente do quadro de lesão das fibras cardíacas por um processo hipóxico-isquêmico (MINICUCCI et al., 2010).

Com base nos dados literários não observamos nenhuma pesquisa que correlacione o efeito da fotobiomodulação com quadro de enfisema instalado e sua repercussão extrapulmonar a nível enzimático. Então levantamos a seguinte hipótese: a fotobiomodulação diminuirá os níveis de enzima $\mathrm{CK}_{\mathrm{MB}}$ na corrente sanguínea.

Esta hipótese pode ser comprovada, pois observamos que havia diminuição dos níveis de $\mathrm{CK}_{\mathrm{MB}}$, com resultado estatisticamente significativo $(p \leq 0,05)$ quando comparamos os grupos GD e GDL com o controle. Além de observarmos um resultado ainda mais significativo, quando comparamos o grupo GD e GDL onde houve diminuição da enzima no grupo GDL $(p=0,009)$ (Figura 4). Esta hipótese pode ser respaldada pelo fato de que a fotobiomodulação diminui os níveis de interleucinas, como a IL-6, que regula a expressão de outras citocinas inflamatórias como a IL-1 e o Fator de Necrose Tumoral (TNF- $\alpha$ ) (GÓRSKA; KRENKE, 2010), sendo responsável pela liberação de $\mathrm{CK}_{\mathrm{MB}}$ na corrente sanguínea.

\section{CONCLUSÃO}

Com base nos resultados obtidos pode-se concluir que a fotobiomodulação por meio do laser de baixa potência diminui o processo inflamatório, pois diminui as células inflamatórias que desencadeiam o quadro de inflamação extrapulmonar, neste caso, no sistema cardíaco com diminuição concomitantemente da enzima $\mathrm{CK}_{\mathrm{mb}}$ diminuindo, assim, os riscos de infarto agudo. Entretanto, são necessários novos estudos com outras enzimas e citocinas para ratificar o estudo realizado.

\section{REFERÊNCIAS}

BARNES, P. J.; CELLI, B. R. Systemic manifestations and comorbidities of COPD. Eur Respi J, v. 33, n. 5, p. 11651185, 2009.

CAMPOS, H. S. Asma e DPOC: duas faces de um mesmo dado. J. bras. med, v. 103, n. 1, 2015.

CEZARE, T. J. et al. Doença pulmonar obstrutiva crônica. RBM rev. bras. med, v. 72, n. 5, 2015.

CASTARDELI, E. et al. A exposição crônica à fumaça do cigarro resulta em remodelação cardíaca e prejuízo da função ventricular em ratos. Arq Bras Cardiol, v. 84, n. 4, p. 320-4, 2005.

FUSCO, L. B.; PÊGO-FERNANDES, P. M.; XAVIER, A. M.; PAZETTI, R.; RIVERO, D. H. R. F.; CAPELOZZI, V. L.; JATENE, F. B. Modelo experimental de enfisema pulmonar em ratos induzido por papaína. J Pneumol, v. 28, n. 1, p. 1-7, 2002.

GARCIA, L. F.; MATAVELI, F. D.; MADER,A. M.;THEODORO, T. R.; JUSTO, G. Z.; PINHAL, M. A. Cells involved in extracellular matrix remodeling after acute myocardial infarction. Einstein, v. 13, n. 1, p. 89-95, 2015.

GÓRSKA, K. M. W. M.; KRENKE, R. Airway inflammation in chronic obstructive pulmonary disease. Curr Opin Pulm Med, p. 16:89-96, 2010.

GOLD. Guidelines Global Strategy for Diagnosis, 
Management, and Prevention of COPD. Disponível em: <http://www.goldcopd.org/uploads/users/files/ GOLDReport.april112011.pdf $>$. Acesso em: 30 ago. 2014.

HANSSON, J.; VASAN, R. S.; ÄRNLÖN, J.; INGELSSON, E.; LIND, L.; LARSSON, A.; MICHAËLSSON, K.; SUNDSTRÖM, J. Biomarkers of extracellular matrix metabolism (MMP9 and TIMP-1) and risk of stroke, myocardial infarction, and cause-specific mortality: cohort study. PLoS One, v. 6, n. 1, p. 16185, 2011.

ICOCHEA, A.; COOPER, B. S.; KUHN, C. The effect of oxigen on cor pulmonale in experimental emphysema induced by elastase or elastase and betaaminopropionitrile in hamsters. Am Rev Respir Dis, v. 126, n. 5, p. 792-796, 2010.

KARU, T. I. Primary abd secondary mechanisms of action of visible to near-IR irradiation on cells. J Photochem Photobiol B., v. 49, p. 1-17, 1999.

KUHL, C. P. Desenvolvimento de diferentes graus de enfisema pulmonar induzido por elastase em camundongos. 2013. 84f. Dissertação (Mestrado em Ciências Cirúrgicas) - Universidade Federal do Rio Grande do Sul, Faculdade de Medicina, Porto Alegre, 2013.

KOZMA, R. L. H. Modelo experimental de indução de enfisema pulmonar por exposição à fumaça de cigarro. 2012. 67f. Dissertação (Mestrado em Biotecnologia) - Instituto Butantan, São Paulo, 2012.

LIAO, Z. G. et al. Effect of multi-combination absorption enhancer on puerarin intestinal absorption and its toxicity $[\mathrm{J}]$. Chinese Traditional and Herbal Drugs, v. 7, p. 1099-1103, 2012.

MINICUCCI, M. F. et al. Relação entre a Esfericidade, a Função Ventricular e o Tamanho do Infarto em Ratos. Arq bras cardiol, v. 94, p. 645-650, 2010.

MONTEIRO, R. Avaliação das alterações anatômicas cardíacas secundárias ao enfisema pulmonar: estudo experimental em ratos. 2004. 102f. Tese (Doutorado em Cirurgia Torácica e Cardiovascular) - Universidade de São Paulo, São Paulo, 2004.
OLIVEIRA JÚNIOR, G. C. G. C.; EVANGELISTA DO SANTO, L. A.; PEREIRA DE SÁ, H.; MOURA NUNES, H.; SANTOS ALVES, W.; CASTRO CARVALHO, K. Efeito da laserterapia de baixa potência na inflamação pulmonar em rattus novergicus. Conscientiae saúde (Impr.), v. 9, n. 4, 2010.

PRICE, D.; YAWN, B.; BRUSSELLE, G.; ROSSI, A. Relação risco-benefício dos corticosteroides inalatórios em pacientes com DPOC. Prim Care Respir J, v. 22, n. 1, p. 92-100, 2013.

RIBEIRO, B. G.; ALVES, A. N.; SANTOS, L. A. D.; CANTERO, T. M.; FERNANDES, K. P. S.; DIAS, D. S.; BERNARDES, N.; DE ANGELIS, K.; MESQUITA-FERRARI, R. A. Red and Infrared Low-Level Laser Therapy Prior to Injury with or without Administration after Injury Modulate Oxidative Stress during the Muscle Repair Process. PloS one, v. 11, n. 4, p. e0153618, 2016.

RIBEIRO, A. G.; COTTA, R. M.; RIBEIRO, S. M. R. A Promoção da Saúde e a Prevenção Integrada dos Fatores de Risco para Doenças Cardiovasculares The Promotion of Health and Integrated Prevention of Risk Factors for Cardiovascular Diseases. Ciênc saúde coletiva, v. 17, n. 1, p. 7-17, 2012.

RUFINO, R.; DA COSTA, C. H. Patogenia da doença pulmonar obstrutiva crônica. Revista Hospital Universitário Pedro Ernesto, v. 12, n. 2, 2013.

SULKOWSKA, M.; MUSIATOWICZ, B.; SULKOWSKI, S.; ZIMNOCH, L.; EJSMONT-PIETROW, G.; SULIK, M.; DZIECIOL, J.; FAMULSKI, W.; POCZOPKO, B. Cor pulmonale in experimental lung emphysema. I. The morphometric evaluation of pulmonary and myocardium changes. Rocz Akad Med Bialymst., v. 40, n. 2, p. 335343, 1994.

SEIMETZ, M. et al. Inducible NOS inhibition reverses tobacco-smoke-induced emphysema and pulmonary hypertension in mice. Cell, v. 147, n. 2, p. 293-305, 2011.

SMITH, B. M. et al. Pulmonary hyperinflation and left ventricular mass. Circulation, p. CIRCULATIONAHA. 113.001653, 2013.

SILVA, J. C. Avaliação do uso do laser de baixa 
potência e droga fotossensibilizadora no processo de cicatrização. 2003. 100f. Tese de Doutorado. Dissertação (Mestrado em Bioengenharia) - Instituto de Pesquisa e Desenvolvimento, Universidade do Vale do Paraíba, 2003.

TARANTINO, A. B. Doenças pulmonares. Guanabara Koogan, 2008.

VALENÇA, S. S.; PORTO, L. C. Estudo imunohistoquímico do remodelamento pulmonar em camundongos expostos à fumaça de cigarro. J. bras. pneumol., v. 34, n. 10, p. 787-95, 2008.

Recebido: 16 de dezembro de 2015

Revisado: 24 de abril de 2016

Aceito: 01 de maio de 2016 